\title{
Efeitos de Procedimento de Distração Não Contingente em Tratamento Odontopediátrico
}

\author{
Leatrice Palieraqui Pereira da Silva \\ Antônio Bento Alves de Moraes \\ Gustavo Sattolo Rolim \\ Universidade Estadual de Campinas
}

\begin{abstract}
RESUMO
O objetivo deste trabalho foi avaliar se um procedimento de distração não contingente, concomitante ao atendimento, facilitaria o manejo de comportamentos, aumentando a colaboração das crianças. Neste estudo, avaliaram-se dois participantes (PI e PII) de 4 anos, caracterizadas como não colaboradoras. A estratégia de distração realizada na cadeira odontológica ocorreu em sistema de perguntas e respostas não contingente aos comportamentos da criança, baseadas em figuras, ao longo das sessões. As respostas corretas eram reforçadas socialmente e por entrega de fichas-prêmios. As treze sessões foram filmadas e os comportamentos registrados a cada 15 segundos. Observaram-se, comportamentos de protesto, manifestados por meio de alta frequência de choro (PI:1s-74\%, 7s-35\%, PII: 1s-94\%, 6s-40\%), apontando que a distração não contingente não apresentou resultados significativos na redução da frequência de respostas não colaborativas. No entanto, o desempenho de acerto de respostas mediante distração aumentou com o decorrer das sessões (PI: $2 \mathrm{~s}-29 \%$ e $7 \mathrm{~s}-100 \%$ de acerto, PII: 2 s- $25 \%$ e $6 \mathrm{~s}-100 \%$ de acerto). Pode-se considerar que a distração não se revelou como uma estratégia adequada com os sujeitos estudados, porém permitiu que estes realizassem as tarefas planejadas, que não eram incompatíveis com não colaboração. A criança aprende outros repertórios mesmo quando submetida a situações aversivas.
\end{abstract}

Palavras-chave: manejo do comportamento; distração não contingente; psicologia aplicada à saúde.

\begin{abstract}
Non Contingent Distraction Procedure Effects in Pediatric Dentistry Treatment

The aim of the study was to investigate if a non-contingent distraction procedure, during the treatment, would facilitate behavior management increasing the children's cooperation. In this study, two four-year old children, presenting non-compliance to the treatment were evaluated. The distraction technique carried out in dental chair, during the session, involved questions and answers based in pictures. Correct answers were socially reinforced through chips and prizes. Thirteen sessions were recorded and the behaviors analyzed in each 15 seconds intervals. Indicative uncooperative behaviors expressed by high crying frequency (PI:1s-74\% and 7s-35\%, PII:1s-94\% and 6s-40\%) suggests that this non-contingent distraction was not effective in reducing children's uncooperative behaviors. However, children's distraction task was improved during the treatment (PI:2s-29\% and $7 \mathrm{~s}-100 \%$ of correct answers, PII:2s-25\% and 6s-100\% of correct answers. Among the subjects of this study, distraction did not modify non-cooperative behavior, but allowed children to participate of the distraction planned task, which was not incompatible with non-cooperation responses. The child learned other behaviors at the same time as being exposed to aversive conditions.

Keywords: behavior management; distraction; pediatric dentistry.
\end{abstract}

Crianças conduzidas a sessões de atendimento à saúde por seus pais e/ou responsáveis, dependendo de suas condições físicas, podem ser expostas a potenciais procedimentos invasivos, incluindo situações de dor, real ou potencial, e/ou desconforto físico e psi- cológico. Estas situações constituem oportunidades para que crianças e acompanhantes possam adquirir e manter comportamentos mais eficientes para enfrentar o tratamento, especialmente se forem atendidos por profissionais de saúde treinados para a utilização de 
estratégias não aversivas de manejo comportamental e cognitivo (Moraes, Costa Junior \& Rolim, (2004).

Em odontologia, o cirurgião-dentista que se dispõe a tratar da saúde bucal de crianças deve manter-se atualizado no que se refere à aplicação de uma variedade de estratégias que auxiliem seus pacientes a lidarem com diferentes demandas do tratamento odontológico. Antes e durante o tratamento, o profissional deve estar apto a identificar os principais indicadores do desenvolvimento da criança, analisando os comportamentos apresentados durante a exposição ao ambiente de cuidados odontológicos.

A criança que apresenta patologias dentais e baixa colaboração ao tratamento odontológico, constitui um objeto potencial de pesquisa, estimulando os profissionais a buscarem estratégias psicológicas de adesão ao tratamento e de manejo de comportamentos considerados não colaborativos com as rotinas odontológicas a serem executadas. Por meio de sistemas eficientes de comunicação, profissionais de saúde podem aliviar o medo e a ansiedade, antecipada e concomitante, ao tratamento, ensinar mecanismos de enfrentamento apropriados e orientar a criança a tornar-se mais cooperativa, relaxada e confiante no papel de paciente em contexto odontológico (American Academy of Pediatric Dentistry, 2008).

Como vários procedimentos odontológicos, especialmente aqueles de caráter curativo, podem gerar respostas de medo e ansiedade em grande parte dos pacientes e, em especial, entre as crianças (Costa Junior, 2002), a utilização de procedimentos de distração, por exemplo, simultaneamente à realização de sessões de tratamento odontológico, pode facilitar o manejo comportamental dos pacientes, contribuindo para o aumento do grau de colaboração com a execução das rotinas odontológicas e reduzindo o tempo de exposição da criança ao consultório. Há mais de 20 anos, a Organização Mundial de Saúde (World Health Organization, 1983) já recomendava a distração como uma estratégia potencialmente útil aos profissionais de saúde, particularmente em contextos que envolvessem a submissão de pacientes a procedimentos invasivos.

Procedimentos de distração podem ser considerados como exemplos de estratégia de incentivo à mudança do foco de atenção do paciente, do procedimento odontológico para outros estímulos percebidos como mais agradáveis. Por exemplo, iniciar alguma atividade lúdica como jogos ou videogame, enquanto o profissional executa determinado procedimento, ou, como destaca Angelotti (2004) "tudo que requeira alguma forma de atenção e que faça o paciente desviar-se do estímulo original".

Segundo Stark e cols. (1989), a distração é uma estratégia proveitosa, pois os fatores geradores da distração são estímulos que podem obter algum controle sobre as respostas colaborativas do paciente, que se tornam incompatíveis com o comportamento não colaborador.

Peretz e Gluck (2005) compararam a efetividade de um procedimento de distração (um truque de mágica) em relação à técnica de conte-mostre-faça. As crianças, de três a seis anos de idade, caracterizadas como resistentes ao tratamento, durante a primeira visita odontológica, foram divididas em dois grupos: o de distração e o de controle. Os resultados demonstram mudanças significativas nos comportamentos das crianças que participaram do grupo de mágica, reduzindo-se o tempo decorrido entre a entrada e o início do atendimento e o tempo necessário para tomadas radiográficas.

Dados obtidos por Peretz e Gluck (2005), permitem apontar que, algumas vezes, estratégias relacionadas à transmissão de informação podem constituir eventos potencialmente aversivos e que estratégias de distração podem aumentar a probabilidade de que a criança enfrente, de modo mais relaxado e colaborativo, contextos percebidos anteriormente como estressantes e ansiogênicos.

Wilson e Cody (2005) realizaram um estudo de revisão sobre estratégias de manejo comportamental, referidas em dois periódicos especializados (Pediatric Dentistry e Journal of Dentistry for Children) nos últimos 30 anos, e sobre a extensão das evidências empíricas destas técnicas em odontopediatria. Encontraram 168 estudos, sendo 30\% sobre descrições clínicas, $38 \%$ em levantamentos de opiniões e $32 \%$ baseados em questionários e descrições de uso das estratégias de manejo. Observaram, também, que na última década, ocorreu uma redução na publicação de artigos com relatos baseados em evidência de pesquisa que sustentam as vantagens do uso do manejo comportamental em odontopediatria. Com relação às estratégias de distração, os autores, descrevem que os estudos são, tipicamente, distrações verbais utilizadas durante a aplicação de anestesia, em detrimento de outras modalidades como desenhos infantis, vídeos e músicas, utilizadas de modo mais esporádico e nem sempre contingentes ao desempenho comportamental dos pacientes.

As evidências clínicas e de pesquisa sugerem que a distração contingente ao desempenho comportamental 
de pacientes, mediada por princípios de reforçamento positivo, são mais efetivas que a utilização de estratégias não contingentes. No entanto, tais estudos ainda requerem alguns refinamentos metodológicos, incluindo, entre outros, análises funcionais de comportamentos, registro observacional sequencial, definição operacional de comportamentos e análise de respostas em vídeo (Aitken, Wilson, Coury \& Moursi 2002; Ingersoll, Nash, Blount, \& Gamber, 1984).

Frente às demandas clínicas e de pesquisa em saúde, considera-se que a investigação de estratégias não aversivas de manejo, que concorram com a exposição de crianças a eventos potencialmente dolorosos ou desconfortáveis, e não contingentes ao repertório de comportamentos das mesmas, constitua um objeto relevante de pesquisa. Acredita-se que dados obtidos a partir de tais procedimentos possam contribuir com a geração de conhecimentos mais sistemáticos sobre o uso e os efeitos de estratégias de distração sobre o repertório de comportamento de crianças expostas a tratamentos odontológicos.

O objetivo deste estudo foi analisar os efeitos de um procedimento de distração não contingente sobre o repertório de comportamentos não colaborativos de crianças submetidas a tratamentos odontológicos. Pretendeu-se avaliar se o procedimento em questão concorre com os eventos potencialmente aversivos de algumas rotinas odontológicas, reduzindo a ocorrência de comportamentos de resistência ao tratamento.

\section{MÉTODO}

\section{Participantes}

Participante I (PI): menino com 4 anos e 2 meses, acompanhado pelo irmão ou mãe durante as sessões de tratamento. Encaminhado para atendimento de emergência por motivo de dor de dente. Verificou-se que a criança apresentava cavidades de cárie nos elementos $84,85,75,74,55,54$ e 53 , que necessitavam de restauração e tratamento endodôntico. Foi executada, então, uma adequação bucal, sem necessidade de aplicação de anestesia injetável e agendada a primeira sessão de tratamento.

Participante II (PII): menina com 4 anos e 3 meses, acompanhada pela mãe durante as sessões. A condição bucal apresentava cavidades de cárie nos dentes 55, 85, 75, 74, 64, 84, com indicação de restaurações e tratamento endodôntico.

A seleção dos participantes foi realizada durante a primeira sessão de atendimento odontológico, ao exame clínico. Foram selecionadas aquelas que apresentassem níveis moderados e leves de respostas não colaborativas com o tratamento, tais como protestos verbais, movimentos do corpo e cabeça e choro.

Os responsáveis pelas crianças selecionadas foram informados sobre os objetivos da pesquisa e convidados a assinar o Termo de Consentimento Livre e Esclarecido. O projeto de pesquisa foi aprovado pelo Comitê de Ética em Pesquisa (CEP) sob o Protocolo 096/2004.

\section{Procedimento}

A sessão inicial de atendimento foi utilizada como linha de base de comportamentos dos participantes.

Sessões experimentais de distração não contingente: o procedimento consistiu na formulação de questões verbais concomitantes à execução das rotinas odontológicas e respostas das crianças por meio de gestos manuais. As questões permitiam duas modalidades de resposta: gesto manual com o polegar para cima (sinal positivo, indicativo de "sim") ou com o polegar para baixo (sinal negativo, indicativo de "não"). As questões se referiam ao conteúdo de pôsteres que retratavam desenhos ou situações cotidianas e que estavam afixados acima da cadeira odontológica.

As questões eram formuladas aleatoriamente e em momentos não especificados, isto é, não definidos previamente e nem associados à determinada rotina odontológica, e também não estavam relacionados a quaisquer comportamentos dos participantes, colaborativos ou não colaborativos com o tratamento. Desta forma, da segunda sessão em diante (com um total de sete sessões para PI e seis sessões para PII) a estratégia de distração foi realizada concomitantemente o tratamento.

Antes do início do atendimento foi realizado um treino de respostas com as crianças, familiarizando-as com o procedimento. Durante o treino, as crianças eram instruídas a observar um pôster e a responder questões utilizando os gestos manuais. As figuras apresentadas em treino eram diferentes das figuras utilizadas nas sessões de tratamento. Ainda durante o treino, os participantes foram informados que receberiam uma ficha a cada resposta correta e que dependendo do número de fichas obtidas, poderiam, ou não, trocá-las por um brinquedo ao final de cada sessão ou de um conjunto de sessões. Todas as respostas corretas das crianças foram reforçadas socialmente com elogios. Após o treino, iniciaram-se as sessões semanais de tratamento e a aplicação do procedimento de 
distração não contingente. Todas as sessões de tratamento foram realizadas pela mesma cirurgiã-dentista $\mathrm{e}$ auxiliar.

Registro dos comportamentos não colaboradores: Todas as sessões foram gravadas em vídeo para posterior observação sistemática e análise dos comportamentos dos participantes. As gravações receberam sinais sonoros a cada 15 segundos (bipes), sendo nestes momentos realizado o registro dos comportamentos das crianças conforme as categorias comportamentais propostas por Possobon (2003).

\section{RESULTADOS}

Os resultados do presente estudo estão apresentados em tabelas com as frequências relativas dos comportamentos não colaborativos e as respostas de acerto, erro e não respostas relacionadas ao procedimento de distração não contingente, divididas por sessão e por rotina.

Os dados apresentados na Tabela 1 ilustram as frequências das respostas não colaborativas dos Participantes I e II, em cada sessão de atendimento odontológico e as frequências relativas dos acertos, erros e não respostas às questões referentes ao procedimento de distração não contingência.

TABELA 1

Frequência de Respostas Não Colaborativas (Nc) e das Respostas de Acerto, Erro e Não Resposta dos Participantes I e II em Cada Sessão

\begin{tabular}{|c|c|c|c|c|c|c|c|c|c|c|}
\hline & & Tempo & & & & & & & & \\
\hline & & (min) & $\mathrm{Re}$ & $\mathrm{Rc}$ & $\mathrm{Cn}$ & $\mathrm{R}$ & $\mathrm{Ch}$ & erro & acerto & não resposta \\
\hline \multirow[t]{7}{*}{ P1 } & 1 sessão & 43 & - & - & $1 \%$ & $3 \%$ & $74 \%$ & - & - & - \\
\hline & 2 sessão & 41 & - & - & $1 \%$ & $1 \%$ & $72 \%$ & $48 \%$ & $29 \%$ & $24 \%$ \\
\hline & 3 sessão & 33 & - & - & - & $4 \%$ & $63 \%$ & $24 \%$ & $71 \%$ & $6 \%$ \\
\hline & 4 sessão & 61 & - & - & - & - & $22 \%$ & $5 \%$ & $84 \%$ & $11 \%$ \\
\hline & 5 sessão & 31 & - & - & - & $6 \%$ & $45 \%$ & $15 \%$ & $65 \%$ & $20 \%$ \\
\hline & 6 sessão & 26 & - & - & $2 \%$ & $7 \%$ & $58 \%$ & - & $88 \%$ & $13 \%$ \\
\hline & 7 sessão & 24 & - & - & - & $4 \%$ & $35 \%$ & - & $100 \%$ & - \\
\hline \multirow[t]{5}{*}{ P2 } & 1ạ sessão & 12 & - & - & $9 \%$ & $6 \%$ & $94 \%$ & - & - & - \\
\hline & 2a sessão & 36 & - & $7 \%$ & $8 \%$ & - & $86 \%$ & $6 \%$ & $25 \%$ & $69 \%$ \\
\hline & 3a sessão & 23 & - & $3 \%$ & $2 \%$ & $3 \%$ & $96 \%$ & - & $73 \%$ & $27 \%$ \\
\hline & 5ạ sessão & 31 & $2 \%$ & - & $2 \%$ & $2 \%$ & $95 \%$ & $11 \%$ & $89 \%$ & - \\
\hline & 6a sessão & 73 & $3 \%$ & - & - & $2 \%$ & $40 \%$ & - & $100 \%$ & - \\
\hline
\end{tabular}

Re: Recusa; Rc: Resistência a Contenção; R: Reclamação; Cn - Comportamento Nervoso; Ch: choro

Observa-se que PI apresentou, durante os atendimentos, as seguintes respostas, Choramingo (Cn), Reclamação (R) e Choro (Ch). No decorrer das sessões, os comportamentos de protestos apresentaram redução na frequência de ocorrência, especialmente da primeira para a quarta sessão. Todavia, não é possível afirmar que houve aumento gradual de frequência, em termos gerais, dos comportamentos colaborativos. $\mathrm{Na}$ quarta sessão, por exemplo, a criança adormeceu, fato que contribuiu para a baixa frequência de ocorrência de todos os comportamentos (apenas $22 \%$ total de não colaboração). Na segunda e terceira sessões, os comportamentos não colaborativos foram superiores a $65 \%$ do tempo total das sessões. Já na quinta e sétima sessão observou-se novamente redução da frequência de comportamentos não colaborativos (com $51 \%$ e $39 \%$ de não colaboração, respectivamente). Os comportamentos de choramingo e gritos, os mais frequentes, ocorreram em percentuais equivalentes ou superiores a $45 \%$ do tempo da primeira a terceira e da 
quinta e sexta sessões. Desconsiderando-se a quarta sessão, apenas na última, a frequência de choro foi inferior a $40 \%$ do tempo, conforme ilustrado na Tabela 1 .

No decorrer das sessões de tratamento de PII, os comportamentos de protesto não apresentaram redução significativa na frequência de respostas, exceto na última sessão quando os comportamentos não colaborativos foram reduzidos para menos de $45 \%$ (Tabela 1). Comparando-se a sessão de linha de base com a última sessão de tratamento, a frequência de respostas de choro e reclamação foi reduzida para menos da metade. Todavia, não é possível afirmar que houve aumento na frequência de comportamentos colaborativos, uma vez que a frequência de comportamentos não colaborativos de cada sessão foi semelhante, ocorrendo redução de frequência apenas na última sessão, conforme observado na Tabela 1.

Os acertos às questões formuladas para este Participante (PI) estiveram acima de $70 \%$ nas quatro primeiras sessões. A criança vinha reduzindo o nível de não colaboração e aumentando a proporção de acertos desde o início do tratamento. No entanto, na quinta sessão a frequência de não colaboração voltou a aumentar e, consequentemente os acertos foram reduzidos para $65 \%$ das questões, conforme o observado na Tabela 1.

Os acertos de PII estiveram acima de $70 \%$ em três sessões. Ressalta-se que em duas dessas sessões (terceira e quarta) a criança apresentou alto nível de não colaboração, enquanto que na segunda sessão observou-se uma frequência de não colaboração mais baixa. A partir da terceira sessão, o percentual de acertos aumentou progressivamente até a última sessão, quando atingiu $100 \%$, conforme o observado na Tabela 1 .

Os dados apresentados na Tabela 2 se referem às respostas das crianças para as perguntas da pesquisadora. Há três tipos de respostas: certas (a), erradas (e) e ausência de resposta para as perguntas (nr). As rotinas odontológicas, por sua vez, foram divididas conforme a ordem de ocorrência: entrada $(E)$, anestesia tópica (AT), anestesia infiltrativa (AI), isolamento absoluto (IA), preparo cavitário (PC) e restauração (R).

TABELA 2

Frequência das Respostas de Acerto, Erro e Não Resposta dos Participantes I e II por Rotina em Cada Sessão de Atendimento

\begin{tabular}{|c|c|c|c|c|c|c|c|c|c|c|c|c|c|c|c|c|c|c|c|}
\hline \multicolumn{20}{|c|}{ Participante 1} \\
\hline \multicolumn{8}{|c|}{ Acerto } & \multicolumn{6}{|l|}{ Erro } & \multicolumn{6}{|c|}{ Não resposta } \\
\hline Sessões & $\begin{array}{l}\text { Total de } \\
\text { perguntas }\end{array}$ & $\mathrm{E}$ & AT & $\mathrm{Al}$ & IA & PC & $\mathrm{R}$ & E & AT & $\mathrm{Al}$ & $\mathrm{IA}$ & PC & $\mathrm{R}$ & $E$ & AT & $\mathrm{Al}$ & IA & $\mathrm{PC}$ & $\mathrm{R}$ \\
\hline 2 a & 21 & & $5 \%$ & $10 \%$ & - & - & $14 \%$ & $5 \%$ & - & $5 \%$ & $5 \%$ & $5 \%$ & $29 \%$ & - & $5 \%$ & - & - & $14 \%$ & $5 \%$ \\
\hline $3 \underline{a}$ & 17 & - & - & $6 \%$ & $6 \%$ & $35 \%$ & $24 \%$ & - & - & $12 \%$ & - & $6 \%$ & $6 \%$ & - & - & - & - & $6 \%$ & - \\
\hline $4 \underline{a}$ & 19 & - & $5 \%$ & $11 \%$ & $26 \%$ & $37 \%$ & $5 \%$ & - & $5 \%$ & - & - & - & - & - & - & - & $11 \%$ & - & - \\
\hline 5 & 20 & - & - & $5 \%$ & $5 \%$ & $5 \%$ & $50 \%$ & $10 \%$ & - & - & - & - & $5 \%$ & - & $5 \%$ & - & - & $10 \%$ & $5 \%$ \\
\hline $6 \underline{a}$ & 16 & $6 \%$ & $6 \%$ & $19 \%$ & $6 \%$ & $19 \%$ & $31 \%$ & - & - & - & - & - & - & - & - & $6 \%$ & $6 \%$ & - & - \\
\hline 7a & 16 & $6 \%$ & $6 \%$ & $6 \%$ & $19 \%$ & $25 \%$ & $38 \%$ & - & - & - & - & - & - & - & - & - & - & - & - \\
\hline Total & & $2 \%$ & $4 \%$ & $9 \%$ & $10 \%$ & $19 \%$ & $27 \%$ & $3 \%$ & $1 \%$ & $3 \%$ & $1 \%$ & $2 \%$ & $7 \%$ & $0 \%$ & $2 \%$ & $1 \%$ & $3 \%$ & $6 \%$ & $2 \%$ \\
\hline \multicolumn{20}{|c|}{ Participante 2} \\
\hline \multicolumn{8}{|c|}{ Acerto } & Erro & & \multicolumn{10}{|c|}{ Não resposta } \\
\hline Rotinas: & & $\mathrm{E}$ & AT & $\mathrm{Al}$ & IA & $\mathrm{PC}$ & $\mathrm{R}$ & $E$ & AT & $\mathrm{Al}$ & $\mathrm{IA}$ & PC & $\mathrm{R}$ & $E$ & AT & $\mathrm{Al}$ & IA & PC & $\mathrm{R}$ \\
\hline $2 \underline{a}$ & 16 & $6 \%$ & $6 \%$ & $6 \%$ & - & $6 \%$ & - & - & - & $6 \%$ & - & - & - & - & $13 \%$ & $13 \%$ & $25 \%$ & $13 \%$ & $6 \%$ \\
\hline 3 & 11 & - & - & - & $9 \%$ & $9 \%$ & $55 \%$ & - & - & - & - & - & - & $9 \%$ & - & - & - & $9 \%$ & $9 \%$ \\
\hline 5 a & 19 & $5 \%$ & $5 \%$ & $37 \%$ & $16 \%$ & $21 \%$ & $5 \%$ & - & - & $11 \%$ & - & - & - & - & - & - & - & - & - \\
\hline 6a & 34 & $3 \%$ & $3 \%$ & $6 \%$ & $3 \%$ & $74 \%$ & $12 \%$ & - & - & - & - & - & - & - & - & - & - & - & - \\
\hline Total & & $4 \%$ & $4 \%$ & $12 \%$ & $7 \%$ & $27 \%$ & $18 \%$ & $0 \%$ & $0 \%$ & $4 \%$ & $0 \%$ & $0 \%$ & $0 \%$ & $2 \%$ & $3 \%$ & $3 \%$ & $6 \%$ & $5 \%$ & $4 \%$ \\
\hline
\end{tabular}

Na Tabela 2, observa-se que a ocorrência de acertos pelas crianças estava relacionada à rotina odontológica específica. Para PI, o percentual de acertos foi menor nas rotinas iniciais $(\mathrm{E}=2 \%, \mathrm{AT}=4 \% ; \mathrm{AI}=9$,
IA $=10 \%)$ e aumentou gradativamente até o final da sessão ( $\mathrm{PC}=19 \%$ e $\mathrm{R}=27 \%$ ), sugerindo que as duas últimas rotinas eram os momentos menos estressantes para a criança. Para PII, os acertos em cada rotina 
odontológica (Tabela 2), também, estavam relacionados às rotinas específicas. Nas últimas rotinas, os percentuais de acerto foram maiores, principalmente durante o Preparo Cavitário ( $\mathrm{PC}=27 \% ; \mathrm{R}=18 \%$ ).

\section{DISCUSSÃO}

Nesta seção discute-se a efetividade do procedimento de distração não contingente sobre os comportamentos não colaborativos e o desempenho, em termos de respostas corretas, para o procedimento executado.

Observam-se, comumente, em contextos de tratamento odontológico respostas de medo e ansiedade, identificados por meio de padrões de não colaboração com as exigências do tratamento, ou seja, comportamentos de esquiva e fuga (Moraes e cols., 2004). Em nosso estudo observou-se que as crianças apresentaram indicadores comportamentais de esquiva e protesto, manifestados por meio de alta frequência de respostas de choro alto, gritos e choramingo (Tabela 1). Observou-se também, padrões de protesto verbal que, provavelmente, estavam relacionados aos eventos aversivos, mantidos pela situação de atendimento (Tabela 1, por exemplo, PI nas sessões 5 e 6 e PII nas sessões 3 e 5).

Os resultados sugerem que a técnica de distração, da forma como foi empregada, apresentou efeitos sobre a redução de comportamentos, conforme demonstrado na Tabela 1. Porém, não é possível inferir que estes padrões de não colaboração foram eliminados ou que a distração proporcionou uma adaptação ou um enfrentamento mais eficiente da situação. $\mathrm{Na}$ última sessão de atendimento, PI e PII apresentaram padrões de respostas comportamentais de protesto, observando-se sua manutenção no decorrer das sessões. Na última sessão de tratamento, ambos os participantes apresentaram comportamentos de não colaboração ( $42 \%$ e $39 \%$, respectivamente), o que não permite inferir que a distração teve efeito significativo sobre a colaboração.

Resultados semelhantes foram obtidos por estudos que também empregaram diferentes técnicas de distração em procedimentos médicos ou odontológicos com crianças (Stark e cols., 1989; Sullivan, Schneider, Musselman, Dummet Jr, \& Gardiner, 2000; Carlson, Broome e Veesey, 2000; Marwah, Prabhakar e Raju, 2005; Cassidy e cols., 2002; Aitken e cols., 2002). Algumas hipóteses podem ser levantadas para os resultados obtidos nestes estudos, tais como: (1) a exposição da criança por períodos longos de tempo a pro- cedimentos invasivos; (2) a falta de percepção de controle da criança durante a exposição à situação de tratamento odontológico; (3) a repetição do estímulo de distração ao longo das visitas, o que sugere o desenvolvimento de habituação a tal condição; (4) a interrupção ou atraso dos procedimentos odontológicos em função de comportamentos não colaborativos da criança ao mesmo tempo em que participava do procedimento de distração, provavelmente, permitiu que a criança aprendesse a tarefa solicitada e não colaborasse com o tratamento.

No entanto, outros autores demonstram a eficácia de procedimentos de distração em contextos de tratamento de saúde (Patel e cols., 2006; Peretz \& Gluck, 2005; Dahlquist, Pendley, Landthrip, Jones \& Steuber, 2002). As hipóteses levantadas para os resultados destes estudos incluem: (1) o tipo de distração empregada envolvia o uso de diferentes modalidades sensoriais, tais como tato, visão e audição, ao mesmo tempo; (2) o tipo de atividade envolvida (ativa ou passiva); (3) o estabelecimento de vínculo entre paciente e profissional durante a atividade de distração aumentava a confiança da criança no profissional.

No presente estudo, pode-se considerar que as respostas de protesto verbal, apresentadas por PI, segundo a Escala de Comportamentos de Stark e colaboradores (1989), estão relacionadas a um padrão não colaborativo leve (classificado como escore 4). Segundo esta classificação, os comportamentos não colaborativos leves incluem: (a) choro ou resmungos que gerem a necessidade de interrupção do procedimento, com o fim de acalmar a criança; (b) apresentações repetidas de instruções pelo dentista; e (c) necessidade de ajuda física para a realização dos procedimentos de rotina. No entanto, apesar do padrão apresentado por PI, ter sido considerado como um comportamento não colaborativo, o choro não impediu a realização dos procedimentos planejados. Já para PII, o padrão comportamental apresentado poderia ser avaliado pela Escala como não colaborador moderado (escore 5), pois os movimentos de corpo e cabeça foram, em vídeo, identificados como mais frequentes, requerendo, ocasionalmente, o uso de contenção física (Tabela 1).

O padrão de choro frequente de PI e PII relacionam-se às seguintes variáveis:

(1) repetição das rotinas de tratamento odontológico ao longo das sessões, que podem ter gerado uma resposta condicionada de antecipação de protestos da criança. Por exemplo, até a rotina de anestesia infil- 
trativa os acertos dos dois participantes foram baixos; levando-se em consideração que a quantidade de perguntas formuladas nessas rotinas também foi reduzida (em função dos comportamentos de protesto das crianças), este dado é coerente com a de Cassidy e cols. (2002), nos quais as agulhas foram apontadas pelas crianças como os estímulos que mais provocavam ansiedade e que mais se associavam as experiências dolorosas em ambiente hospitalar e de tratamento de saúde em geral;

(2) muitos acertos do procedimento de distração ocorreram na presença de choro, que pode ter sido reforçado junto com as respostas corretas (o comportamento selecionado conjugava duas classes de respostas, responder corretamente às perguntas e chorar, isto é, a criança era reforçada positivamente no momento choro-acerto);

(3) a ausência da liberação de reforçamentos positivos, por parte do dentista, contingente a comportamentos colaborativos da criança e na ausência de choro; segundo Schiff, Holtz, Peterson e Rakusan (2001), o reforçamento positivo liberado mediante comportamentos considerados adequados tem o objetivo de recompensar a criança pelo esforço em determinadas tarefas. Por exemplo, ficar quieta durante a execução de um procedimento que envolve algum desconforto físico e receber um incentivo social imediatamente depois da apresentação de comportamentos considerados adequados. Deste modo, espera-se um aumento da probabilidade de que a criança adquira e mantenha estratégias eficientes de enfrentamento quando for exposta, novamente, às mesmas contingências.

Outra possível explicação se deve ao fato do choro ser considerado como uma resposta típica de crianças expostas a procedimentos odontológicos invasivos. Stokes e Kennedy (1980), por exemplo, realizaram um estudo com técnicas de modelação e reforçamento para redução de comportamentos não colaborativos de crianças submetidas a tratamento odontológico. Para os autores, crianças jovens submetidas a tratamento odontológico exibem, frequentemente, uma série de comportamentos não colaborativos, tais como movimentos agitados e choro. Nesse contexto, Shinohara e cols. (2005) realizaram um estudo para avaliar a relação entre os comportamentos de crianças $(n=33)$, entre três e nove anos de idade, durante tratamento odontológico. Dados obtidos apontaram que pacientes que exibem medo e ansiedade antes do tratamento, tendem a apresentar atitudes não colaborativas durante o tratamento, destacam-se a recusa a submeter-se ao tratamento, o choro, as manifestações verbais de medo e os movimentos físicos (mexer as mãos, colocar as mãos sobre a boca, espernear), que atrasam a execução do tratamento.

Ressalta-se que, em nosso estudo, o dentista foi instruído a não utilizar qualquer técnica de manejo para redução de comportamentos de choro e os acompanhantes também receberam instruções para interferir o mínimo possível sobre o comportamento das crianças. No estudo de Stark e cols. (1989), por exemplo, os participantes recebiam reforçamento positivo apenas quando acertavam as respostas. Levanta-se a hipótese de que se acompanhantes e dentista tivessem sido instruídos a reforçar as respostas corretas da criança e os comportamentos colaborativos ao longo das sessões, poder-se-ia esperar maior ocorrência de comportamentos colaborativos, redução da frequência e choro e obtenção de $100 \%$ de acertos das perguntas em momento anterior ao obtido (PI e PII obtiveram $100 \%$ de acerto apenas na última sessão de tratamento).

No entanto, deve-se observar que comportamentos não colaborativos de protesto verbal se mantiveram ao longo das sessões e que as respostas de choro não foram concorrentes ao desempenho da distração, ou seja, a criança poderia protestar, chorar e acertar as perguntas simultaneamente. Estes resultados corroboram os dados do estudo de Stark e colaboradores (1989), no qual as crianças aprenderam, após repetidas exposições à distração, que elas poderiam se engajar em comportamentos não colaborativos e responder a um número suficiente de perguntas para receber o prêmio.

Os participantes deste estudo (PI e PII) participaram do procedimento de distração à medida que eram expostas às sessões de tratamento odontológico, apresentando desempenho gradativamente maior, expresso pelo aumento da frequência de respostas corretas e redução da frequência de choro em determinadas sessões (Tabelas 1 e 2).

Mesmo em situações potencialmente aversivas ocorre alguma forma de aprendizagem, ou seja, mesmo sob contingências aversivas, que produzem ou eliciam respostas de protesto verbal, PI não apresentou respostas de fuga ou esquiva ou repostas indicadoras de medo, e PII apresentou respostas de recusa em duas sessões. Os participantes aprenderam uma nova classe de respostas: acerto, erro ou não resposta, quando uma classe de estímulos (perguntas) era disponibilizada, em uma situação em que estavam submetidas a condições aversivas. Os dados deste estudo sugerem a valorização desta condição, na qual algu- 
mas respostas estão sob controle de situações aversivas e uma nova condição, disposta pelo pesquisador, concorrente à contingência aversiva, permite que a criança adquira um padrão de respostas que leva à redução de comportamentos não colaborativos com o tratamento. Destaca-se que esta nova condição não implica em supressão de respostas; o choro foi mantido, por eliciação de respostas e/ou ganho de suporte a presença de um acompanhante, e a resposta de acerto foi estabelecida de forma efetiva ao longo das sessões. Estas respostas, choro e acerto, não são incompatíveis, ou seja, podem ocorrer de forma concomitante. Funcionalmente, o choro é mantido pela situação e a resposta de acerto é mantida pelo ganho posterior, o brinquedo, após o encerramento da sessão.

Algumas limitações deste estudo podem ter favorecido os resultados encontrados: (a) o próprio desenho do estudo, que se baseou em uma distração não contingente, ou seja, acontecia a qualquer momento, desta forma, é possível que tenhamos selecionado comportamentos indesejados dos participantes ao reforçar o desempenho positivo para a distração; (b) devido à própria técnica de distração escolhida para o estudo, que pode não ter oferecido estímulo suficiente para, além de engajar a criança na atividade, mudar o foco de atenção do tratamento em execução e reduzir o medo da criança a ponto de fazê-la parar de chorar.

\section{CONSIDERAÇÕES FINAIS}

Para ambos os participantes, as repostas de não colaboração mantiveram-se no decorrer do tratamento odontológico, exceto na última sessão, quando a frequência de não colaboração foi reduzida. No entanto, PI apresentou menor nível de não colaboração quando comparado com PII. É provável que esta criança tenha se adaptado à situação de tratamento e não que o procedimento de distração, na última sessão de tratamento, tenha influenciado a redução da frequência de comportamentos não colaborativos.

Os resultados do presente estudo apontam que a distração, da forma como foi empregada, não apresentou resultados significativos na redução dos comportamentos não colaborativos das crianças. Todavia, a literatura disponível nesta área ainda apresenta resultados inconclusivos e a distração constitui uma técnica promissora no manejo de comportamentos não colaborativos com tratamentos de saúde e na redução da ansiedade de pacientes odontopediátricos, sendo necessário, ainda, o desenvolvimento de estudos nesta área.

\section{REFERÊNCIAS}

Aitken, J. C., Wilson, S., Coury, D., \& Moursi, A. M. (2002). The effect of music distraction on pain, anxiety and behavior in pediatric dental patients. Pediatric Dentistry, 24, 114-118.

American Academy of Pediatric Dentistry (2008). Guideline on behavior guidance for the pediatric dental patient. Retirado em 08 de outubro de 2008, de http://www.aapd.org/search/default.asp.

Angelotti, G. (2004). Distração. Em C. N. Abreu \& H. J. Guilhardi (Org.), Terapia comportamental e cognitivo-comportamental: Práticas clínicas (pp. 393-397). São Paulo: Roca.

Carlson, K. L., Broome, M., \& Vessey, J. A. (2000). Using distraction to reduce reported pain, fear and behavioral distress in children and adolescents: A multiside study. Journal for Specialists in Pediatric Nursing, 5(2), 75-85.

Cassidy, K. L., Reid, G. J., McGrath, P. J., Finley, G. A., Smith, D. J., Morley, C., Szudek, E. A., \& Morton, B. (2002). Watch needle, watch TV: Audiovisual distraction in preeschool immunization. Pain Medicine, 3(2), 108-118.

Costa Junior, A. L. (2002). Psicologia aplicada à odontopediatria: Uma introdução. Estudos e Pesquisas em Psicologia, 2(2), $67-$ 77.

Dahlquist, L. M., Pendley, J. S., Landthrip, D. S., Jones, C. L., \& Steuber, C. P. (2002). Distraction intervention for preschooler undergoing intramuscular injections and subcutaneous port access. Health Psychology, 21(1), 94-99.

Ingersoll, B. D., Nash, D. A., Blount, R. L., \& Gamber, C. (1984). Distraction and contingent reinforcement with pediatric dental patients. Journal of the American Dental Association, 51, 203207.

Moraes, A. B. A., Costa Junior, A., \& Rolim, G. S. (2004). Medo do dentista: Ainda existe?. Em M. Z. S. Brandão (Org.), Sobre comportamento e cognição: Vol. 14 - Estendendo a psicologia comportamental e cognitiva aos contextos da saúde, das organizações, das relações pais e filhos e das escolas (pp. 171178). Santo André: Esetec.

Patel, A., Schieble, T., Davidson, M., Tran, M. C. J., Schoenberg, C., Delphin, E., \& Bennet, H. (2006). Distraction with a handheld video game reduces pediatric preoperative anxiety. Pediatric Anesthesia, 16, 1019-1027.

Peretz, B., \& Gluck, G. (2005). Magic trick: A behavioural strategy for the management of strong willed children. International Journal of Paediatric Dentistry, 15, 429-436.

Possobon, R. F. (2003). Avaliação dos comportamentos de crianças não-colaboradoras, durante o atendimento odontológico, na vigência do ansiolítico diazepan. Tese de Doutorado nãopublicada, Universidade Estadual de Campinas, Piracicaba/SP.

Schiff, W. B., Holtz, K. D., Peterson, N., \& Rakusan, T. (2001). Effect of an intervention to reduce procedural pain and distress for children with HIV infection. Journal of Pediatric Psycho$\log y, 26(7), 417-427$.

Stark, L. J., Allen, K. D., Hurst, M., Nash, D. A., Rigney, B., \& Stokes, T. F. (1989) Distraction: Its utilization and efficacy with children undergoing dental treatment. Journal of Applied Behavior Analysis, 22(3), 297-307. 
Sullivan, C., Schneider, P. E., Musselman, R. J., Dummet Jr, C. O., \& Gardiner, D. (2000) The effect of virtual reality during dental treatment on child anxiety and behavior. Journal of Dentistry for Children, 67, 193-196.

Shinohara, S., Nomura, Y., Shingyouchi, K., Takase, A., Ide, M., Moriyasu, K., Idaira, Y., Takahashi, T., Yamada, Y., Aoyagi, Y., \& Asada, Y. (2005). Structural relationship of child behavior and its evaluation during dental treatment. Journal of Oral Science, 47(2), 91-96.

Stokes, T. F., \& Kennedy, S. H. (1980). Reducing child uncooperative behavior during dental treatment through modeling and reinforcement. Journal of Applied Behavior Analysis, 13(1), 41-49.

Wilson, S., \& Cody, W. (2005). An analysis of behavior management papers published in the pediatric dental literature. Pediatric Dentistry, 27(4), 331-338.
World Health Organization (1983). Behavioural sciences: Preparation for invasive procedures. Geneva: World Health Organization.

Marwah, N., Prabhakar, A. R., \& Raju, O. S. (2005). Music distraction: Its efficacy in management of anxious pediatric dental patients. Journal of Indian Society of Pedodontics and Preventive Dentistry, 23(4), 168-170.

\section{Nota:}

${ }^{1}$ Financiamento CAPES/CNPq

\section{Sobre os autores:}

Leatrice Palieraqui Pereira da Silva: Cirurgiã-Dentista. Mestranda do Programa de Pós-graduação em Saúde da Criança e do Adolescente - Faculdade de Ciências Médicas / Universidade Estadual de Campinas.

Antônio Bento Alves de Moraes: Professor Titular da Faculdade de Odontologia de Piracicaba / Universidade Estadual de Campinas.

Gustavo Sattolo Rolim: Psicólogo, doutorando do Programa de Pós-graduação em Saúde da Criança e do Adolescente - Faculdade de Ciências Médicas / Universidade Estadual de Campinas.

Endereço para correspondência: Antônio Bento Alves de Moraes - Faculdade de Odontologia de Piracicaba / UNICAMP Av. Limeira, 901 - 14414-903 Piracicaba / SP. Endereço eletrônico: abento@fop.unicamp.br. 